und stelle ein zweites Nicol, in einem Theilkreise drehbar, vor dem am Apparat befestigten zwischen dissem und der Lampe auf. Dann stelle man diesen zweiten Nicol so ein, dass er nur wenige Grade von der Stellung entfernt ist, in der er beide Farben ganz auslöscht, stelle den am Apparal befestigten Nicol so, dass Blau merklich heller als Roth erseheint und steigern dann durch Drehung des Nicols der Lampe zunächst die Helligkeit beider Farben in gleichem Masse. Hierbei wird zunächst das Ueberwiegen der Helligkeit für das Blau abnehmen und schliesslich Roth und Blau gleich hell, unter Umständen sogar Roth heller als Blau erscheinen. So kann man zeigen, dass, wenn man die Lichtstärke von Roth und Blau in gleichem Verhältniss steigert, die scheinbare Helligkeit des Roth schneller zunimmt, als die des Blau.

Beide beschriebenen Apparate werden von den Herrn Franz Schmidt und Haenseh in Berlin angefertigt.

In einer nächsten Arbeit denke ich einige Versuche, die ich mit beiden Apparaten angestellt habe, mitzutheilen.

Berlin, den 22. November 1880.

\title{
Beiträge zur Lehre von der Arsenikwirkung auf den thierischen Organismus.
}

Von

\section{J. Dogiel.}

Hierzu Tafel II und 1 Holzschnitt.

Die vorliegenden Untersuchungen iiber die Arsenikwirkung auf den thierischen Organismus beziehen sich: 1) auf das Verhalten des Arsenigsäure-Anhydrids, $\mathrm{As}_{2} \mathrm{O}_{3}$, den Eiweisskörpern gegeniiber, 2) auf seine Veränderungen im Organismus, 3) auf die quantitative Bestimmung des Arsenik in der Leber nach seiner 
Beiträge z. Lehre v. d. Arsenikwirk. auf d. thierischen Organismus. 329

Verabfolgung dem lebenden Thiere per os und 4) auf die Veränderungen, welche der Arsenik in der Blutcirculation hervorruft. Meine Versuche stellte ich an Hunden, Kaninchen und Fröschen an, welche bald mit Arsenigsäure-Anhydrid (Acidum arsenicosum), $\mathrm{As}_{2} \mathrm{O}_{3}$, bald mit Arsensäure (Acidum arsenicicum), $\mathrm{H}_{3} \mathrm{AsO}_{4}$, bald endlich mit Kali- oder Natronsalzen der genannten Säuren vergiftet wurden.

Bevor wir zu den Versuchen selbst übergehen, finde ich es für nöthig hier einige Eigenschaften des Arsenigsäure-Anhydrids und der Arsensäure in Erinnerung zu bringen. Die Wirkung des Arsenigsäure-Anhydrids und anderer Arsenverbindungen hängt theilweise von den chemischen und physikalischen Eigensehaften dieser Stoffe $a b$. Aus diesem Grunde war $_{g}$ ich auch gezwungen meine Aufmerksamkeit zuerst den Eigenschaften des Arsenik und seiner Verbindungen zuzuwenden. Da aber die physikalische und chemische Beschaffenheit des Arsenik und seiner Sauerstoffverbindungen schon ziemlich gut bekannt sind, so brauche ich hier diejenigen Eigenschaften des Arsenigsäure-Anhydrids und der Arsensäure hervorzáheben, welche für meine Untersuchungen von besonderem Interesse sind. $\mathrm{Za}$ den Eigenschaften, welche die eine Arsenverbindung von der anderen unterscheiden lassen, gehören ihre Löslichkeit in destillirtem Wasser und ihr Verhalten den Eiweisskörpern gegenüber. Das Arsenigsäure-Anhydrid ist in Wasser sehr schwer löslich. Man kennt es in zwei Formen: als glasige und als porcellanartige Masse. Aus letzterem Zustand kann es durch anhaltendes Kochen in den ersteren übergeführt werden; bei niedriger Temperatur geht hingegen die glasartige Masse in die porcellanartige über.

Beim Befeuchten des Pulvers vom glasartigen ArsenigsäureAnhydrid mit Ammoniak kann man eine geringe Temperaturerböhung nachweisen. Erwärmt man das verkäufliche ArsenigsäureAnhydrid mit Ammoniakflüssigkeit bis auf $80^{\circ} \mathrm{C}$, so scheiden sich aus der erkalteten Lösung oktaëdrische Krystalle aus. Das Arsenigsäure-Anhydrid krystallisirt im regulären System, oder in rhombischen Prismen. Beim Uebergang des porcellanartigen Arsenigsäure-Anhydrids in die glasartige Form ändert sich nicht nur sein Ansehen, sondern auch sein Löslichkeitsgrad im Wasser. Dieses Verhalten muss bei den Bestimmungen der Löslichkeit des Arsenigsäure-Anhydrids überhaupt beriucksichtigt werden, wie 
man aus nachfolgenden Angaben über die Löslichkeitsverbältnisse entnehmen kann.

\begin{tabular}{|c|c|c|c|}
\hline $\begin{array}{c}\mathrm{Nr} \text {. der } \\
\text { Beobach- } \\
\text { tung. }\end{array}$ & 1 Theil $\mathrm{As}_{2} \mathrm{O}_{3}$. & $\begin{array}{l}\text { Wieviel } \\
\text { Theile } \\
\text { kochen- } \\
\text { den } \\
\text { Wassers. }\end{array}$ & Name des Beobachters. \\
\hline $\begin{array}{l}1 . \\
2 . \\
3 . \\
4 . \\
5 . \\
6 . \\
7 . \\
8 . \\
9 . \\
10 . \\
11 . \\
12 . \\
13 . \\
14 . \\
15 .\end{array}$ & 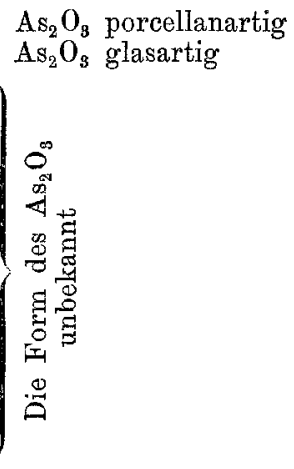 & $\begin{array}{c}7.72 \\
9.33 \\
10.5 \\
11.34 \\
12.0 \\
12.2 \\
15.0 \\
16.0 \\
21.0 \\
24.0 \\
40.0 \\
64.0 \\
80.0 \\
200.0 \\
610.0\end{array}$ & $\begin{array}{l}\text { Guibourt } \\
\text { Wenzel } \\
\text { Fischer } \\
\text { Klaproth } \\
\text { Buchholz } \\
\text { Brandt, Justi, Bergmann } \\
\text { R. A. Vogel } \\
\text { Taylor } \\
\text { Lemétherie } \\
\text { Pöner } \\
\text { Baumé } \\
\text { Nadier } \\
\text { Nase } \\
\text { Haglen. }\end{array}$ \\
\hline
\end{tabular}

Ein Theil $\mathrm{As}_{2} \mathrm{O}_{3}$ scheint vom kalten, warmen oder kochenden Wasser verschiedene Mengen zur Lösung zu bedürfen.

\begin{tabular}{|c|c|c|c|c|}
\hline 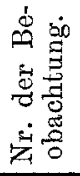 & 1 Theil $\mathrm{As}_{2} \mathrm{O}_{3}$. & $\mid \begin{array}{c}\text { Temperatur des } \\
\text { Wassers. }\end{array}$ & 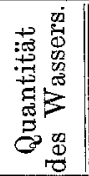 & $\begin{array}{c}\text { Name } \\
\text { des Beobachters. }\end{array}$ \\
\hline $\begin{array}{l}1 . \\
2 . \\
3 . \\
4 . \\
5 . \\
6 . \\
7 . \\
8 . \\
9 . \\
10 . \\
11 . \\
12 . \\
13 .\end{array}$ & $\begin{array}{l}\left\{\begin{array}{c}\mathrm{As}_{2} \mathrm{O}_{3} \text { von unbe- } \\
\text { kannter Form }\end{array}\right. \\
\mathrm{As}_{2} \mathrm{O}_{3} \text { porcellanartig } \\
\mathrm{As}_{2} \mathrm{O}_{3} \text { glasartig } \\
\left\{\begin{array}{c}\mathrm{As}_{2} \mathrm{O}_{3} \text { von unbe- } \\
\text { kannter Form }\end{array}\right. \\
\mathrm{As}_{2} \mathrm{O}_{3} \text { glasartig } \\
\mathrm{As}_{2} \mathrm{O}_{3} \text { porcellanartig }\end{array}$ & $\left\{\begin{array}{c}\text { (kaltes Wasser ? } \\
+10^{\circ} \\
+35^{\circ} \\
+20^{\circ} \\
\text { kaltes Wasser? } \\
+13^{\circ} \\
+13^{\circ} \\
+13^{\circ} \mathrm{C} . \\
+100^{\circ} \mathrm{C} .\left(5^{\prime}\right. \\
\text { hierauf abge- } \\
\text { kühlt) } \\
+100^{\circ} \mathrm{C} .\left(60^{\prime}\right. \\
\text { hierauf abge- } \\
\text { kühlt) }\end{array}\right.$ & \begin{tabular}{|c||}
50 \\
66 \\
80 \\
80 \\
103 \\
96 \\
96 \\
320 \\
420 \\
25 \\
83 \\
59.5 \\
71.8 \\
\\
35.1
\end{tabular} & $\begin{array}{l}\text { Buchholz } \\
\text { Fischer } \\
\text { Bergmann } \\
\text { Guibourt } \\
\text { Spielmann } \\
\text { Hahnemann } \\
\text { Nasse } \\
\text { Klaproth } \\
\text { Bussy } \\
\text { M. Sajzew } \\
\text { und } \\
\text { J. Dogiel. }\end{array}$ \\
\hline
\end{tabular}

Aus diesen Beobachtungen kann sehr leicht entnommen wer* den, wie verschieden der Löslichkeitsgrad des Arseniksäure-Anhy- 
Beiträge z. Lehre v. d. Arsenikwirk auf d. thierischen Organismus. 331

drids in Wasser aufgefasst wird. Diese Meinungsverschiedenheit lässt sich wahrscheinlich dadurch erklären, dass die Versuche nicht unter gleichen Bedingungen ausgeführt sind. $\mathrm{Zu}$ diesen Bedingungen gehören: 1) die Form des Arsenigsäure-Anhydrids; 2) die Temperatur des Wassers; 3) die Daner des Auslangens oder Kochens von $\mathrm{As}_{2} \mathrm{O}_{3}$ mit Wasser und 4) der Grad der Abkühlung der Lösung.

Wie Taylor annimmt, löst kaltes Wasser, wenn es auf einige Stunden mit pulverisirtem Arsenigsäure-Anhydrid in Berührung gebracht wird, vom letzteren von $1 / 1000$ bis $1 / 500$ seines eigenen Gewichts; eine Stunde lang gekocht, hält das Wasser nach dem Erkalten bis ${ }^{1 / 40}$ seines eigenen Gewichts vom ArsenigsäureAnhydrid in Lösung.

Die Gegenwart organischer Stoffe im Wasser ist dem Auflösen des weissen Arsenik hinderlich. Die wässerige Lösung des weissen Arsenik ist durchsichtig, farblos und von schwach saurer Reaction. Kocht man weissen Arsenik mit Wasser weniger als eine Stunde, so löst sich von ihm nicht mehr als $1 / 80$. Eine Unze warmen Thees, mit Milch and Zucker versetzt, oder eine Unze kalten Porters löst nur 1/2 gr vom weissen Arsenik; in einer Unze heissen Caffee, wie in einer Unze kalten Branntweins löst sich $1 \mathrm{gr}$ anf. Im Aether ist er unlöslich. In Fetten ist das ArsenigsäureAnhydrid sehr wenig löslich, nämlich in 1000 Theilen Fett löst sich ungefähr 0,6 von $\mathrm{As}_{2} \mathrm{O}_{3}$. Im Glycerin ist $\mathrm{As}_{2} \mathrm{O}_{3}$ leichter löslich als im Wasser (Annales therapeutiques 1862). Mehr als im Wasser löst sich die arsenige Säure im Chlorwasserstoff. Das Arsenigsäure-Anhydrid löst sich leicht in den Lösungen von alkalischen und kohlensauren Salzen.

Die arsenigsauren Salze sind ein-, zwei- oder dreibasisch. Die Alkalisalze der arsenigen Sätre sind in Wasser löslich und krystallisirbar. Aehnlich der Salpetersäure wirkt eine wässrige Lösung der arsenigen Säure auf eine Lösung von Hühnereiweiss nicht ein. Kocht man die Lösung des Arsenigsäure-Anhydrids (erhalten durch Kochen von $\mathrm{As}_{2} \mathrm{O}_{3}$ im Wasser während einer Stunde) mit gleicher Menge von Hühnereiweiss, welches mit etwas Wasser versetzt war, so erhält man eine weisse Trübung und dann ein Eiweisscoagulum, welches durch Wasserzusatz nicht verändert wird.

Arsensäure $\mathrm{H}_{3} \mathrm{AsO}_{4}$ (acidum arsenicicum) wird aus der arsenigen Säure durch Oxydation vermittelst Salpetersäure oder Königswasser und Abdampfen erhalten. 
In reinem Zustande stellt sie eine durchsichtige, glasartige oder undurchsichtige, weisse, zerfliessliche Masse dar, welche beim Erhitzen in Wasser, Sanerstoff und Arsenigsäure-Anhydrid zerfällt. Leitet man schweflige Säure, $\mathrm{H}_{2} \mathrm{SO}_{3}$, in eine Lösung von Arsensäure, so oxydirt sie in Schwefelsäure, $\mathrm{H}_{2} \mathrm{SO}_{4}$, während die Arsensäure in das Arsenigsäure-Anhydrid ïbergeht. Die Arsensäure löst sich leicht im Wasser. Ihre Salze sind ein-, zwei- oder dreibasisch. Die einbasischen arsensauren Salze sind alle in Wasser löslich und reagiren sauer; von den zwei- und dreibasischen arsensauren Salzen lösen sich in Wasser nur die Alkaliverbindungen und zeigen eine mehr weniger alkalische Reaction. Die Arsensäure macht das Hühnereiweiss nicht gerinnen, auch durch Kochen des Gemisches gerinnt letzteres nicht, es bildet sich jedoch eine geléeartige Masse, welche sich bei Wasserzusatz auflöst. Die geléeartige Masse wird noch leichter vom starken Spiritus (70 nach Tralles) und durch Kochen aufgelöst.

Einführungswege des Arsenik in den thierischen Organismus. Arsenigsäure-Anhydrid, Arsensäure und ihre Salze können durch den Magen, die Lungen, die kranke oder gesunde Hant, den Mastdarm, die Schleimhaut der Vagina, der Nase, der Augen und durch directe Einspritzung ins Blut in den Körper gelangen. Der gewöhnlichste und am häufigsten benutzte Applicationsort ist jedoch der Magen.

Kommt der Arsenik in dieser oder jener Form mit der Schleimhaut des Magendarmcanals in Berührung, so gelangt er nach kürzerer oder längerer Zeit ins Blnt; diese Zeitdauer wird sowohl durch ehemische Eigenschaften der applicirten Arsenikverbindung, wie durch die Beschaffenheit der Schleimhaut des Verdautngstractus bedingt. So ist die Zeit, welche bis zum Eintritt des Arsenik ins Blut verläuft, verschieden, je nachdem man ihn in Pulverform oder in Lösung in den Magen bringt.

Dasselbe gilt von der Arsensäure, obwohl hierbei nicht za vergessen ist, dass sie sich ungleich leichter in Wasser löst als die arsenige Säure. Von den Salzen sind nur diejenigen, welche sich leicht in Wasser lösen, durch die Magendarmschleimhaut resorbirbar: alkalische Salze der arsenigen und Arsensäure.

Das arsenigsaure Kupfer (Scheele's Grün) ist in Wasser unlöslich; führt man es jedoch in den Magen ein, so gelangt es ins Blut in Folge der Einwirkung des Magen- und Darmsaftes. Die 
Beiträge z. Lehre v. d. Arsenikwirk. auf d. thierischen Organismus. 333

Quantität und Qualität des Speisebreies im Magendarmcanal bleibt auch nicht ohne Einwirkung auf die Zeit, in welcher die Aufsaugung des Arsenik aut diesem Wege zu Stande kommt. Hinzuzufügen ist, dass bei der Abschätzung der Schnelligkeit der Arsenikresorption noch die Beschaffenheit der Magendarmschleimhaut und des Gefässsystems zu berücksichtigen ist.

Durch den Mastdarm geht die Aufsaugung des ArsenigsäureAnhydrids und der Arsensäure nach der Meinung vieler Beobachter ungleich schneller und gleichmässiger, als durch den Magen vor sich. Gleiches lässt sich aller Wahrscheinlichkeit nach von dem Dünndarm aussagen. Hierbei verdient der Umstand Beachtung, dass die Gegenwart von Schwefelwasserstoff im Darm zweifellos dem Eintritt des Arsenik ins Blut ein Hinderniss darbietet.

Der Arsenik kann in Form von Dämpfen der arsenigen Säure eingeathmet werden, was gerade nicht selten in Arsenikfabriken stattgefunden hat. Als Beispiel, dass der Arsenik durch die Lungen aufgenommen wird, kann der Umstand dienen, dass Tapezierer und Arbeiter, weløhe mit arsenikhaltigen Tapeten zu thun haben, häufig an Arsenikvergiftung leiden. In der Literatur sind Vergiftungsfälle in Folge der Einathmung von Arsenwasserstoff bekannt geworden. Mir kam ein Fall von Arsenikvergiftung vor in Folge der Behandlung einer Ohrenkrankheit mit Arsenikpräparaten durch Kurpfuscher. Ungleich schneller kann man die Wirkung der arsenigen Säure beobachten, wenn man ihre Lösung unter die Haut oder direct in die Blutbahn injicirt. Schlägt man auch den letzteren Weg nicht ein, wenn es gilt Arsenik dem Menschen zu verabfolgen, so greift man doch bei Thierversuchen nicht selten zu dieser Methode.

Wenn auch eine Aufnahme des Arsenik in den Körper, bei der Application des Arsenigsäure-Anhydrids oder der Arsensäure durch die unverletzte Haut, von einigen Beobachtern für möglich gehalten wird, so kann man sich, meiner Meinung nach, doch eher damit für einverstanden erklären, dass die Resorption des Arsenik vielleicht nur eintritt in Fällen, welchen eine theilweise oder gänzliche Zerstörung der Epithelschichte durch anhaltenden Gebrauch der arsenigen oder Arsensäure oder durch andere Agentien vorhergegangen ist.

Die Wirkung des Arsenik auf den thierischen Organismus. Das metallische Arsen wird kaum eine Wirkung auf 
den Organismus haben; seine Verbindungen mit Sauerstoff und Wasserstoff gehören aber ohne Zweifel zu den stärksten Giften. Diese Verbindungen rufen nicht allein im Organismus des Menschen, der Säugethiere, der Vögel, der Fische und Amphibien und der Insecten starke Veränderungen hervor, sondern wirken sogar auf die Pflanzen ein. Bei einigen Thieren erscheint die Wirkung stärker als bei anderen. Obwohl die Arsenikpräparate auf die Pflanzen eine giftige Wirkung ausüben, wächst doch mucor imperceptibilis in der gesättigten Lösung von arseniger Säure.

Pferde, Schafe und Rinder vertragen ziemlich grosse Gaben von Arsenigsäure-Anhydrid, wie einige Beobachter es angeben. Die Wirkung des Arsenigsäure-Anhydrids und der Arsensäure und ihrer Salze auf den Menschen ist nicht immer gleich, was von vielen Umständen abhängt, wie: Dosis, Gewöhnung, Klima etc.

Boudin ${ }^{1}$ ), weleher die Wirkung der arsenigen Säure an sich selbst erprobte, giebt an, dass nach kleinen Gaben er eine allgemeine Erregung, wie nach dem Genuss starken Caffé's, empfand; es trat eine ungewöhnliche Energie der unteren Extremitäten ein. Zuweilen konnte er eine Zunahme mit nachheriger Abnahme der Kraft und der Zahl der Herzcontractionen bemerken. Buchbeim giebt an, dass nach einer Gabe von $1 / 20-1 / 10$ gr arseniger Säure Magenschmerz, welcher mit Hungergefühl verwechselt wird, auftritt.

Werden kleine Gaben häufig wiederholt oder die Dosis selbst vergrössert, so fühlt man Schmerz in der Magengegend, es tritt Appetitmangel, Uebelkeit, Erbrechen und Durchfall ein. Hierzu gesellt sich Trockenheit der Haut, fieberhafter Zustand, Schlaflosigkeit und Kopfschmerz.

Die ferneren Erseheinungen in Folge des Arsenikgenusses weisen schon auf chronische Vergiftung hin, wie: Affection der unteren Extremitäten (Tabes dorsalis), Durchfall abwechselnd mit Verstopfung, Verdaungsstörungen, heftige Magenschmerzen nach Genuss der leichtverdaulichsten Speisen, Unruhe, Abnahme der Geisteskräfte, Schlaflosigkeit, allgemeine Abmagerung und Hautausschläge und Geschwüre, Ausfallen der Haare und Nägel. Ausserdem hat man Gliederzittern, ungewöhnliche Schwäche, Gliederschmerz, Krämpfe und selbst Tetanus, Beklemmung, Lähmung and - Tod beobachtet.

1) Bou din, Traité des fièvres intermitt. et continues des pays chauds etc. Paris 1842. 
Beiträge z. Lehre v. d. Arsenikwirk. auf d. thierischen Organismus. 335

Meine Untersuchungen äber die Wirkung des Arsenik auf den Organismus bezogen sich, wie angeftbrt, auf die Veränderungen der Bluteirculation unter dem Einfluss des ArsenigsäureAnhydrids, der Arsensäure und ibrer Salze.

Nachdem man einen von den soeben genannten Stoffen, in kleiner Dosis, nach beliebiger Methode, in die Blutbahn eines Thieres (Frosch, Kaninchen, Hund) gebracht, wird eine Beschleunigung der Herzcontractionen bemerkbar; grosse Gaben bewirken eine solche Abänderung der Herzcontractionen; wie man es gewöhnlich bei der Erstickung zu beobachten Gelegenheit hat, es treten hier nämlich Schwankungen der Frequenz, Kraft und des Rhythmus auf, wie es durch nachfolgende Curve ersichtlich wird (Fig. 1).

Die Curve, Fig. I, ist beim Versuch am 680 Gramm sehweren Hunde, dem eirea $6 \mathrm{ccm}$ einer Lösung von $\mathrm{As}_{2} \mathrm{O}_{3}$ in die Schenkelvene eingeführt war, erhalten worden. Die Lösung von $\mathrm{As}_{2} \mathrm{O}_{3}$ war durch Kochen (eine Stunde) und nachheriges Abkühlen des glasartigen Arsenigsäure-Anhydrids im Wasser erhalten und filtrirt worden. Die linke Carotis stand mit dem Manometer des Kymographen von Ludwig in Verbindung. Beim Seltenerwerden der Herzschläge sind diese kräftiger oder auch sehwächer. Die Herz" töne werden ihrerseits anch stärker oder schwächer. Die Zahl der Herzeontractionen bei den Fröschen kann von kleinen Gaben $\mathrm{As}_{2} \mathrm{O}_{3}$ auf 10 Schläge in der Minute zunehmen und auf dieser Höhe ziemlich lange verbleiben.

Bei den Versuchen an Hunden erhält man der Dosirung des Arsenigsäure-Anhydrids entsprechende Veränderungen der Herzcontractionen, nämlich Beschleunigung der Herzschläge, welche nach meinen Beobachtungen auf 180 bis 204 in der Minute steigen kann, zuweilen wurde aber eine Verlangsamung von 180 auf 52 Schläge in der Minute constatirt. Solche starke Schwankungen in der Zahl und der Kraft der Herzeontractionen kamen gewöhnlich nach brechenerregenden Gaben zur Beobachtung. Während des Brechactes tiberhaupt, folglich auch beim Erbrechen in Folge der Arsenikwirkung, tritt eine Verlangsamung der Herzcontractionen ein; nach dem Brechact findet im Gegentheil eine Beschleunigung der Herzeontractionen statt.

Ueberhaupt macht sich die Wirkung kleiner Gaben des Arsenigsäure-Anhydrids, der Arsensäure oder ihrer Salze auf das Herz des 
Mensehen und der Thiere durch Beschleunigung der Herzcontractionen geltend; die Wirkung grösserer Gaben aber im Gegentheil durch Verlangsamung and Störungen im Rhythmus.

Bei acuter Vergiftung durch Arsenigsäure-Anhydxid oder Arsensäure sistirt die Athmang vor den Herzcontractionen. Nach erfolgtem Tode des Thieres sind bald alle Herzböhlen von Blut erfüllt; bald enthält nur die rechte Herzhälfte viel Blut; die linke Herzkammer ist zuweilen vollkommen leer und stark contrahirt. Dieser Wechsel der Blutmenge im Herzen der durch Arsenikpräparate vergifteten Thiere kann theilweise durch den mehr oder weniger schnell erfolgten Tod, theilweise aber durch noch näher zu erforschende Umstände bewirkt sein. Was die nächste Ursache der Beschlennigung oder Verlangsamung der Herzcontractionen in Folge der Arsenikwirkung anbelangt, so werden, meiner Meinung nacb, im Anfange der Hemmungsapparat und hierauf erst die automatischen Herzcentra angegriffen, obgleich man dem Arsenik eine Wirkung auch auf die Muskelelemente des Herzens nicht absprechen kann; wenigstens, wie ich mich überzeugen konnte, nimmt die Kraft der Sceletmuskelcontractionen in Folge der Arsenikwirkung anfangs zu.

Der Blutdruck wird anfangs durch die Wirkung des Arsenigsäure-Anhydrids erhöht; jedoch grosse Gaben irgend eines von den ángegebenen Arsenikpräparaten erniedrigen den Blutdruck schnell; diese Blutdruckschwankungen gehen Hand in Hand mit der Affection des vasomotorisehen Centrums.

Bemerkenswerth ist, dass man die Arsenikwirkung auf die Blutgefässe noch zur Zeit, wann die Atbmung schon eingestellt and die Herzeontractionen sehr schwach sind, oder auch schon aufgehörthaben, beobachten kann.

Fig. II zeigt eine Blutdruckerhöhung bei einem mit einer Lösung von $\mathrm{As}_{2} \mathrm{O}_{3}(12 \mathrm{cem})$ durch Injection in die Schenkelvene vergifteten Hundes. Seine Schenkelarterie war mit dem Kymographen Ludwigs verbunden.

Die Curve weist Schwankungen im Blutdruck nach, nachdem die Athmung schon vollkommen sistirte; obgleich die Herzeontractionen fortdauerten, wurden sie doch allmählich schwächer und börten fast ganz auf, während der Blutdruck constant stieg. Dieselben unter Fig. I u. II angefuhhrten Curven demonstrirea Blutdruckschwankungen, welche mit den sogenannten Athmungswellen 
Beiträge z. Lehre v. d. Arsenikwirk. auf d. thierischen Organismus. 337

verwechselt werden könnten, obwohl das Thier schon lange aufgehört hatte zu athmen und auch keine künstliche Athmung stattfand ${ }^{1}$ ).

Die Geschwindigkeit des Blutstroms nimmt anfangs durch die Wirkung des Arsenigsäure-Anhydrids oder der Arsensäure zu. Hierauf folgt bei fernerer Wirkung Verlangsamung des Blutstroms. Die Geschwindigkeit des Blutstroms wurde vermittelst der Stromuhr bestimmt.

Um die von mir beobachtete Wirkung der Arsenverbindungen auf die Geschwindigkeit des Blutstroms anschaulicher zu machen, wollen wir die Zeit, welche eine und dieselbe Menge Blut zum Durchströmen des Gefässes gebraucht, mit Linien bezeichnen. Vergleichen wir nun die Zeit vor und nach dem Einführen des Arsenigsäure-Anbydrids in den Organismus des Thieres, so erhalten wir die gewïnsehten Data in Bezug auf die Geschwindigkeit des Blutstroms bei den verschiedenen Bedingungen und zu verschiedener Zeit.

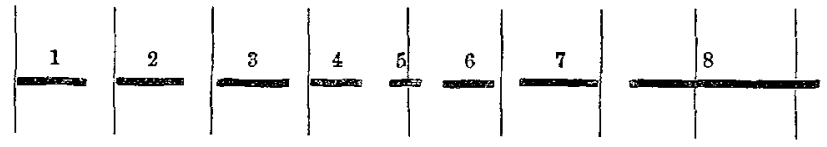

Die vorliegende Zeichnung giebt diese Resultate in Form von Linien; vergleichen wir die Länge der Linien (d. h. die Zeit, welche zum Durchströmen einer und derselben Blutmenge nothwendig ist) unter sich, so sehen wir den Unterschied. Das Arsenigsäure-Anhydrid wurde in die Schenkelvene des Hundes bei Nr. 2 geführt; die Zeit nimmt von Nr. 4 sehon ab, verlängert sich aber hierauf im Vergleich zur Zeit vor der Application des Arsenik um das Dreifache. In Bezug auf die Wirkung des Arsenigsäure-Anhydrids und der Arsensäure auf das Blut selbst, kann ich nur anführen, dass aufgefangenes, defibrinirtes Hunde- oder Froschbiut lange ohne Veränderung bleibt, wenigstens, was die Form der rothen Blutkörperchen und die Blutfarbe betrifft, wenn man dem Blute Arsenigsäure-Anhydrid in Pulverform zusetzt und es sogar einige Tage stehen lässt; setzt man aber zu ebenso defibrinirtem Blute Arsensäure hinzu, so wird die Blutfarbe schnell dunkler und die Form der rothen Blutkörperchen verändert sich:

1) Der Anfang der Curve in Fig. I a. II ist durch $\mathbf{A}$ und $\mathbf{A}^{\prime}$ bezeichnet. 
der Kern des rothen Blutkörperchens (im Froschblut) tritt schärfer hervor und wird körnig.

Um ibber die letzte Ursache der verschiedenen Erscheinungen in Folge der Wirkung der Arsenverbindungen auf den thierisehen Organismus sich annähernden Aufschluss zu verschaffen, muss man uber das Schicksal des Arsenik nach seiner Einführung in den Organismus im Klaren sein.

Die Untersuchung der Veränderungen des Arsenigsäure-Anhydrids und der Arsensäure, nachdem sie mit dem Blut und den verschiedenen Geweben des Organismus in Berührung gekommen. und ihre Ausscheidung ans dem Körper bildet eine der schwierigsten Aufgaben der Pharmakologie. Ein, wenn auch geringer Beitrag in dieser Richtung bleibt, meiner Ansicht nach, nicht ohne Einfluss auf die fernere Bearbeitung dieser Frage, weshalb ich auch meine diesbezuiglichen Beobachtungen mitzutheilen beschloss, ohne das Wesen der Arsenikwirkung auf den thierischen Organismus erklären za wollen.

Die resorbirbaren Arsenverbindungen werden nach ihrer Einführung in den Magen von den Magen-Darmvenen aufgenommen, gelangen in die Leber und von hier aus in den grossen Kreislauf. Doch führt man die eine oder die andere der Arsenverbindungen in den Magen oder direct in die Blutbahn, so tritt Arsenik in der Galle auf, woher er wieder ins Blut gelangen und somit einige Male den Kreislauf passiren kann, bis er auf diese oder jene Weise endlich aus dem Organismus ausgesehieden wird, vorausgesetzt, dass das Thier am Leben bleibt.

Werden die Arsenikverbindungen, indem sie den Weg aus dem Magendarmkanal ins Blut zurücklegen und schliesslich zur Ausscheidung aus dem letzteren gelangen, in ihrer Zusammensetzung verändert oder nicht?

Nach der Meinung Buchbeims verändert sich das Arsenigsäure-Anhydrid, wenn es mit den Bestandtheilen des Organismus in Berührung kommt, obgleich die Art der Veränderung unbekannt bleibt.

Wie Wöhler und Frerichs annehmen, geht die Arsensåure in die arsenige Säure über und erst nach diesem Uebergang zeigt sich eine Wirkung auf dem Organismus. Dieselben Autoren glauben, dass die Arsensäure eine stärkere Wirkung als das Arsenigsäure-Anhydrid besitzt, obgleich Schroff einen Unterschied 
Beiträge z. Lehre v. d. Arsenikwirk. auf d. thierischen Organismus. 339

nicht anerkennt. Buchheim und Sawitseh ${ }^{1}$ ) äusserten den Gedanken, als ob die Wirkung der angegebenen Säuren im directen Verhältniss zur Menge des in ihnen enthaltenen Arsens stehe, was aber Borodin ${ }^{2}$ ) für unrichtig erklärt.

Hannon ${ }^{3}$ ) glaubt dass die arsenige Säure im Magendarmkanal in arsenigsaures Natron uibergeht und in dieser Gestalt ins Blut gelangt, wo mit phosphorsaurem Kalk unlöslicher arsenigsaurer Kalk gebildet wird, welcher sich in den Geweben des Körpers absetzt.

Liebig ${ }^{4}$ ) kam zu dem Schluss, dass die arsenige Säure in und ausser dem Magen mit den Eiweisskörpern eine bestimmte chemische Verbindung bildet. Doch weder Kendall und Edwards ${ }^{5}$ ) noch in letzterer Zeit Binz ${ }^{6}$ ) und Schulz konnten diese Angabe bestätigen.

Meine Untersuchungen sprechen mehr dafür, dass das in den Magen eingeführte und hierauf ins Blut gelangte ArsenigsäureAnhydrid nicht in Arsensäure übergeht; wenigstens ist das Arsenigsäure-Anhydrid, nach der Einfuihrung in den Magen, oder direct in die Blutbahn, einige Zeit als solches im Blute nachweisbar. Die Untersuchungsmethode besteht im Folgenden. Es wurde eine bestimmte Quantität Arsenigsäure-Anhydridlösung in den Magen eingeftihrt. Nach einiger Zeit wurde eine Portion Blut, gewöhnlich aus der Carotis, abgezapft und in einen Dialysator gebracht, dessen zur Aufnahme des Blutes bestimmter Theil aus einem mit englischem Pergament an einem Ende zugebundenen Glascylinder bestand. Dieses Ende stand in einer geringen Menge destillirten Wassers in einem anderen Glasgefäss. Ein Theil der durch die Dialyse aus dem Blute gewonnenen Flïssigkeit wurde zum Nach-

1) R. Buchheim: Lehrbuch der Arzneimittellehre. 3.Aufl. S.309. 1878. - Meletemata de acidi arsenicosi efficacia. Dissertat. inaug. Dorpat 1854.

2) Borodin: Die Analogie der Arsensäure mit der Phosphorsäure. Dissert. Petersburg 1858.

3) Hannon: Revue med. çhirurg. 1849.

4) Liebig: Die Chemie in ihrer Anwendung auf Agricultur und Physiologie. 1843.

5) London pharm. journ. Lex. 1850.

6) C. Binz und H. Schulz: Die Arsengiftwirkungen vom chemischen Standpunkt betrachtet. Archiv für experim. Pathologie und Pharmakologie. XI. Bd. 3. Heft. S. 200. 
weis des Arsens, vermittelst des March'schen Apparates, verbraucht, der andere Theil diente zur Bestimmung, ob man es mit arsenigsauren oder arsensauren Verbindungen zu thun hatte. Den Unterschied festzustellen erlauben bestimmte Reactionen dieser oder jener Verbindung. Zu solchen auch bei meinen Versuchen zur Anwendung gelangten Reactionen wären folgende zu zählen.

Eine Lösung salpetersauren Silbers, $\mathrm{AgNO}_{3}$, mit einigen Tropfen Ammoniak, erzeugt in einer Fliissigkeit, welche arsenige Säure enthält, einen gelben aus arsenigsaurem Silber bestehenden, im Ueberschuss von Ammoniak sich wieder auflösenden Niederschlag. Aus der wässrigen Lösung von Arsensäure erhält man durch $\mathrm{AgNO}_{3}$ einen ziegelrothen Niederschlag von arsensaurem Silber.

Um den etwaigen Antheil phosphorsauren Silbers, $\mathrm{Ag}_{3} \mathrm{PO}_{4}$, an der Bildung des gelben, in der dem Dialysator entnommenen Flüssigkeit durch Zusatz von salpetersaurem Silber and Ammoniak entstehenden Niedersehlag nicht zu übersehen, verglich ich die durch $\mathrm{AgNO}_{3}$ bewirkten Niederschläge in Flüssigkeiten, welche vor und nach der Vergiftung eines Thieres mit Arsenigsäure-Anhydrid, aus dessen Blute durch Dialyse gewonnen waren.

Ausser dieser Reaction auf Arsenigsäure-Anhydrid gebrauchte ich noch Schwefelwasserstoff, $\mathrm{H}_{2} \mathrm{~S}$, welcher bekanntermassen eine reine wässrige Lösung von Arsenigsäure-Anhydrid gelb färbt. Kurze Zeit darauf erscheint ein gelber Niederschlag durch die Bildung von $\mathrm{As}_{2} \mathrm{~S}_{3}$, welcher leicht in Kalihydrat, Ammoniak und Schwefelalkalien, nicht aber in Salzsäure löslich ist.

Nicht überflüssig ist es hier anzuführen, dass ich beim Nachweis des Arsenigsäure-Anhydrids oder der Arsensäure im Blute mich nicht etwa mit einer, aus der Chemie bekannten Reaction begnügte, sondern eine Reaction durch die andere zu controliren suchte.

Die Anwendung des Dialysators finde ich im vorliegenden Falle für nützlich, weil die durch denselben erhaltene Flüssigkeit fast ungefärbt erscheint und deshalb sich zum Nachweis von arsenigsauren oder arsensauren Verbindungen durch die oben angeführten Reactionen eignet. Als Beispiele will ich einige solcher Versuche mittheilen.

Versuch A. Aus der Carotis eines kleinen, jungen Hundes wurde eine Portion Blut entnommen. Hierauf führte man dem Hunde $40 \mathrm{ccm}$ einer 
Beiträge z. Lehre v. d. Arsenikwirk. auf d. thierischen Organismus. 341

durch einstündiges Kochen erhaltenen, wässrigen Lösung von ArsenigsäureAnhydrid in den Magen und unterband den Oesophagus. Nach einiger Zeit traten alle Vergiftungssymptome, Brechanfälle etc. auf. Anderthalb Stunden nach der Einführung des Arsenigsäure-Anhydrids in den Magen des Thieres wurde wieder eine Portion Blut der Carotis entnommen, um es auf den Arsengehalt zu prïfen.

Die durch den Dialysator aus beiden Portionen Blutes erhaltenen Flüssigkeiten wurden der Probe unterworfen. Der March'sche Apparat wies in der zweiten Portion Flüssigkeit Arsen nach. Ferner gaben $\mathrm{H}_{2} \mathrm{~S}$ und $\mathrm{ClH}$ derselben Flüssigkeit eine gelbe $\mathrm{SCaO}_{4}+\mathrm{KHO}$ eine grïne Farbe und endlich erzeugte $\mathrm{AgNO}_{3}$ mit einigen Tropfen $\mathrm{NH}_{3}$ einen gelben Niederschlag, der sich im Ueberschuss des Ammoniaks wieder auflöste.

Hiergegen traten in der zweiten Portion Flüssigkeit, welche aus dem vor der Vergiftung des Thieres der Carotis entnommenen Blute gewonnen war, durch Zusatz von $\mathrm{H}_{2} \mathrm{~S}$ mit $\mathrm{ClH}$ keine Veränderungen ein; $\mathrm{AgNO}_{3}$ erzeugte einen weissen Niederschlag, der sich durch vorsichtige Versetzung mit Ammoniak nicht gelb färbte.

Versuch B. Einem mittelgrossen Hunde wurden $200 \mathrm{ccm}$ einer wässrigen Lösung von $\mathrm{As}_{2} \mathrm{O}_{3}$ (0,2 Gramm) in den Magen eingeführt. 2 Stunden und 30 Minuten nach der Vergiftung entnahm man der Carotis eine Portion Blutes, erhitzte bis zur Gerinnung der Eiweisssubstanzen und brachte hierauf in den Dialysator. Weder die durch Dialyse erhaltene Flüssigkeit noch das geronnene Blut liessen einen Arsengehalt erkennen.

Versuch C. Einem mittelgrossen Hund wurde Arsenigsäure-Anhydrid (c. 0.1 Gramm) in wässriger Lösung in die Schenkelvene injicirt. Eine Stunde darauf entnahm man der Carotis eine Portion Blut und ausserdem nach dem erfolgten Tode des Thieres aus der Gallenblase Galle. Im Blutgerinnsel, wie in der Galle konnte mit dem March'schen Apparat in nicht unbedeutendex Menge Arsen constatirt werden. In der durch Dialyse enthaltenen Flüssigkeit wiesen $\mathrm{H}_{2} \mathrm{~S}$ und $\mathrm{ClH}$, ebenfalls $\mathrm{AgNO}_{3}$ und $\mathrm{NH}_{3}$ eine arsenigsaure Verbindung nach.

Versuch D. In das periphere linke Carotisende eines grossen Hundes wurden $30 \mathrm{ccm}$ einer gesättigten Arsenigsäure-Anhydrit geführt. Das periphere Ende der Vena jug. dextra communis wurde zum Auffangen des Blutes benutzt und war deshalb mit einem Glasrohr verbunden, während die vena jug. sinistra communis unterbunden war. Das auf diese Art gewonnene, venöse Blut gerann schnell; worauf es in den Dialysator gebracht wurde. Die in 40 Stunden durch die Dialyse erhaltene Flüssigkeit zeigte entschieden Arsenigsäure-Anhydrid und nicht Arsensäure.

Ich begnügte mich nicht mit dieser Reihe von Beobachtungen, sondern suchte meine Versuche in Bezug auf die Umwandlungen des Arsenigsäure- 
Anhydrids im thierischen Organismus zu modificiren. $\mathrm{Zu}$ diesem Zweck mischte ich das aus dem Gefässsystem des Thieres erhaltene Blut mit einer wässrigen Lösung von Arsenigsäure-Anhydrid und stellte hierauf die Mischung in den Dialysator. Die durch die Dialyse gewonnene Flüssigkeit nntersuchte ich auf Arsen, wie folgt.

$400 \mathrm{~cm}$ defibrinirtes Hundeblut wurden mit $8 \mathrm{~cm}$ durch einstündiges Kochen erhaltener, wässriger Lösung von Arsenigsäure-Anhydrid vermischt. Die durch Dialyse aus der Mischung gewonnene Flüssigkeit zeigte, nach oben beschriebener Methode untersucht, einen Arsengehalt in Form arsenigsaurer Verbindung.

Hinzufügen kann ich, dass mir oft der Nachweis einer arsenigsanren Verbindung in der Pericardialflüssigkeit von Hunden, denen vordem eine Lösung von Arsenigsäure-Anhydrid in den Magen eingeführt worden war, gelang.

Alle angefiihrten Beobachtungen weisen mit einiger Wahrscheinlichkeit darauf hin, dass Arsenigsäure-Anhydrid, in den Magen eingeführt und ins Blut gelangt, in Form von arsenigsaurer aber nicht arsensaurer Verbindung nachweisbar ist. Weiter zeigen dieselben Beobachtungen, dass man nicht immer Arsen im Blute nachweisen kann, wenn auch vorher Arsenigsäure-Anhydrid in den Magen des Thieres eingeführt war, wie Versuch B) es zeigt. Es wird deshalb nothwendig anzunehmen, dass das Arsenigsäure-Anhydrid aus dem Blute in verschiedene Gewebe und Organe tritt und hier kürzere oder längere Zeit verbleibt, wieder ins Blut übergeht etc. bis es ganz ausgesehieden ist, oder bis das Thier verendet. Wahrseheinlich ist auch die Beobachtung Skalosubow's, der Arsenik hauptsächlich im Gehirn und nicht in anderen Organen - Leber, Muskeln - fand, hierdurch zu erklären.

Ist der Arsenik ins Blut gelangt, so kann man ihn in Gehirn, den Lungen, dem Herzen, den Nieren, dem Darm, der Leber, der Milz, den Knochen, dem Blute, dem Harne und in der Galle nachweisen. In der Leber tritt er sogar häufiger als in anderen Organen und Geweben auf und es scheint, als ob hierbei insofern eine Gesetzmässigkeit waltet, als die Leber nur eine bestimmte Quantität Arsenik autnehmen kann. Ein Maximum Arsenik in der Leber tödtet das Thier; erholt es sich, so verringert sich das Maximum, bis es endlich vollständig ausgeschieden ist.

Die einschlägigen Untersuchungen von Dr. Geoghegan ${ }^{1}$ ) zeigen folgendes. „Nimmt man das Durchschnittsgewicht der Leber

1) Alfr. Taylor: Die Gifte. Bd. I. S. 67. 
Beiträge z. Lehre v. d. Arsenikwirk, auf d. thierischen Organismus. 343

zu dreiundeinhalb Pfund an, so war der Totalbetrag des in diesem Organe abgesetzten Arseniks:

\begin{tabular}{|c|c|}
\hline $\begin{array}{c}\text { Stunden nach Beibringung } \\
\text { des Giftes. }\end{array}$ & $\begin{array}{c}\text { Totalgewicht des } \\
\text { Arseniks. }\end{array}$ \\
\hline Nach $51 / 2$ bis 7 Stunden & 0.8 Gran \\
\hline$\Rightarrow \quad 8^{3} / 4$ & 1.2 \\
\hline$n 15$ & 2.0 \\
\hline$" 17$ bis 20 & 1.3 \\
\hline$" 10^{1} / 2$ Tagen & 1.5 \\
\hline$\Rightarrow \quad 14$ & $0.17 \pi$ \\
\hline
\end{tabular}

Folglich beträgt nach Geoghegan das Maximum der Arsenikanhäufung in der Leber bis 2 Gran. Meine Untersuchungen führten zu etwas von diesem Autor abweichenden Resultaten. Es sollen hier einige dieser Versuche Platz finden.

Versuch den 1. März $1871^{1}$ ). Um 11 Uhr 10 Min. wurden einem Hunde mittelst eines Catheters $50 \mathrm{ccm}$ einer wässrigen Lösung von Arsenigsüure-Anhydrid $\left(0,5\right.$ Gramm $\left.\mathrm{As}_{2} \mathrm{O}_{3}\right)$ in den Magen eingeführt. Den Oesophagus unterband man. Nach 5 Minuten heftiger Brechanfall mit starkem Speicheln. Der Hund überlebte 1 Stunde 5 Min. die Beibringung des Arsenigsäure-Anhydrids. Die Section erfolgte den 2. März. Die 291 Gramm schwere Leber wurde vom Blut gereinigt (abgespült) und in zwei Theile zerschnitten. Ein Theil der Leber (131 Gramm) wurde mit Kali chloricum und Chlorwasserstoffsäure in einem Kolben auf dem Wasserbade oxydirt. Der andere, 160 Gramm schwere Theil der Leber wurde mit rauchender Salpetersäure in einer Retorte oxydirt, in deren Hals ein langes, mit einer Waschvorrichtung verbundenes Rohr steckte. Hiernach wurde die Mischung so lange in der Retorte gekocht, bis sich keine gasförmigen Produkte mehr bildeten und die Flüssigkeit fast farblos wurde. Es bildete sich hierbei eine dünne, ungefärbte Fettschicht. Jetzt wurde die Mischung vorsichtig mit kohlensaurem Natron neutralisirt und filtrirt; das Fett entfernte man durch heisses Wasser. Das Filtrat wurde mit Salzsäure angesäuert und an einem warmen Ort lange mit Schwefelwasserstoffgas bearbeitet. Der braune Niederschlag, der sich dabei bildete, wurde auf einem Filter gesammelt, die Flüssigkeit aber wieder unter denselben Bedingungen mit Schwefelwasserstoff behandelt. Der sich abermals bildende braune Niederschlag wurde wieder abfiltrirt und diese Operationen mehrmäls wiederholt. Die ganze Quantität des braunen Niederschlags, sammt den Filtern unterwarf man hierauf der Oxydation durch rauchende Salpetersäure anf dem Wasserbade. Die auf solchem Wege erhaltene, braune, sauer reagirende Flüssigkeit wurde mit Aetznatron gesättigt, aufs Trockne

1) Die unten angeführten Zahlenwerthe über den Arsengehalt der Leber sind vom damaligen Laboranten meines Laboratoriums, jetzigem Professor der Chemie, Glinsky, erhalten worden. 
abgedampft und mit Salpeter und Soda in einem Tiegel geschmolzen. Die farblose, geschmolzene Masse wurde durch Wasser ausgelaugt. Die wässrige Lösung wurde mit Schwefelsäure-Anhydrid bearbeitet, um die Arsensäure zur arsenigen Säure zu reduciren. Den Ueberschuss des Schwefelsäure-Anhydrids entfernte man durch Erwärmen. Hierauf kam die Lösung, nachdem sie mit Salzsäure angesäuert war, zur Bearbeitung mit Schwefelwasserstoff an einem warmen Ort. Der aus $\mathrm{As}_{2} \mathrm{~S}_{3}$ bestehende gelbe Niederschlag wurde abfiltrirt, die Flüssigkeit wieder unter denselben Bedingungen mit Schwefelwasserstoff behandelt, wobei gewöhnlich sich wieder ein Niederschlag von $\mathrm{As}_{2} \mathrm{~S}_{3}$ bildete. Die ganze Menge des Niederschlags und das Filter wurden im Dampfbade getrocknet und gewogen. Durch eine schwache Aetzammoniaklösung löste man hierauf das Dreifach-Schwefelarsen auf dem Filter. Letzterer wurde nach gehörigem Trocknen im Dampfbade wieder gewogen und durch den Gewichtsunterschied die Menge des $\mathrm{As}_{2} \mathrm{~S}_{3}$ bestimmt. Als Resultat dieser quantitativen Arsenbestimmung in der Leber erscheint Folgendes:

1) 131 Gramm Leber nach der ersten Methode behandelt gaben 0.048 Gramm $\mathrm{As}_{2} \mathrm{~S}_{3}$, was genau 0.0386 Grammen $\mathrm{As}_{2} \mathrm{O}_{3}$ entspricht. Folglich müssten 1000 Gramm Leber 0.2946 Gramm $\mathrm{As}_{2} \mathrm{O}_{3}$ enthalten.

2) 160 Gramm Leber gaben, nach der zweiten Methode behandelt 0.695 Gramm $\mathrm{As}_{2} \mathrm{~S}_{3}$, was genau 0.0559 Grammen $\mathrm{As}_{2} \mathrm{O}_{3}$ entspricht. Folglich müssten 1000 Gramm derselben Leber 0.3493 Gramm $\mathrm{As}_{2} \mathrm{O}_{3}$ enthalten.

Wie man also ersieht, ist die Menge des Arsenigsäure-Anhydrids, berechnet auf 1000 Theile nach oben beschriebenen Methoden behandelter Leber, nicht gleich gross. Die Menge des $\mathrm{As}_{2} \mathrm{O}_{3}$, berechnet nach der zweiten Methode ergab auf 1000 Theile Leber . . . . . . . . 0.3493 Gramm Arsenigsäure-Anhydrid, während die Berechnung nach der

ersten Methode . . . . . . . . . . . . . . . 0.2946 " angiebt, folglich beläuft sich der Unterschied auf . . . . 0.0547 ” Wenn der Unterschied auch gering ist, so existirt er doch. Lassen wir ihn unbeachtet, so beträgt der ganze Gehalt der 291 Gramm schweren Iseber $0.0386+0.0559=0.0945$ Gramm $\mathrm{As}_{2} \mathrm{O}_{3}$.

Versuch am 8. März 1871. Es wurden um 1 Uhr 22 Min. einem jungen Hunde $50 \mathrm{ccm}$ einer Lösung von $\mathrm{As}_{2} \mathrm{O}_{3}$ in den Magen eingeführt $\left(1000 \mathrm{ccm}\right.$ Wasser enthielten $\left.10 \mathrm{Gramm} \mathrm{As}_{2} \mathrm{O}_{3}\right)$. Nach 1 Stunde und $38 \mathrm{Min}$. verendete das Thier. Die Section wurde am 2. Mai vorgenommen, ohne dass ein fauler Geruch dabei wahrnehmbar gewesen wäre. Die linke Herzkammer war fast leer, die rechte aber mit Blut überfüllt. Die 133 Gramm schwere Leber wurde dreimal mit Wasser abgespült und auf zwei Bogen Filtrirpapier gebracht. Die Oxydation geschah durch rauchende Salpetersäure in einer mit bekannten Vorrichtungen versehenen Retorte. Den Arsenik bestimmte man in Form von Dreifach-Schwefelarsen. Hierbei konnte festgestellt werden, dass 133 Gramm Leber $0.0742 \mathrm{Gramm} \mathrm{As}_{2} \mathrm{~S}_{3}$, oder $0.0597 \mathrm{Gramm} \mathrm{As}_{2} \mathrm{O}_{3}$ enthielten, folglich auf 1000 Theile Leber 0.4488 Gramm $\mathrm{As}_{2} \mathrm{O}_{3}$ kommen.

Versuch am 1. Juli 1871 . Einem grossen Hunde wurden $100 \mathrm{ccm}$ 
Beiträge z. Lehre v. d. Arsenikwirk. auf d. thierischen Organismus. 345

conc. wässrige Arsenigsäure-Anhydridlösung in den Magen eingeführt. Nach einer Stunde ging er ein. Bei der Section nahm man die $475 \mathrm{Gramm}$ schwere Lieber und unterwarf sie der Oxydation mit rauchender Salpetersäure auf früher beschriebene Weise. Der Arsenik kam als Dreifach-Schwefelarsen zur Bestimmung. 475 Gràmm Leber gaben 0.1713 Gramm $\mathrm{As}_{2} \mathrm{~S}_{3}$, oder 0.13787 Gramm $\mathrm{As}_{2} \mathrm{O}_{3}$, folglich kommen auf 1000 Gramm Leber 0.29025 Gramm $\mathrm{As}_{2} \mathrm{O}_{3}$.

Ein Vergleich der Arsenigsäure-Anhydridmengen in der Hundeleber in allen drei Fällen ergiebt:

\begin{tabular}{|c|c|c|c|}
\hline Versuchszeit & $\begin{array}{l}\text { Gewicht der } \\
\text { Leber }\end{array}$ & $\begin{array}{l}\text { der Totalgehalt der } \\
\text { Leber an } \mathrm{As}_{2} \mathrm{O}_{3}\end{array}$ & $\begin{array}{c}\text { die Menge der } \\
\mathrm{As}_{2} \mathrm{O}_{3} \text { auf } 1000 \\
\text { Theile Leber }\end{array}$ \\
\hline 1. März 1871 & 291 Gramm & 0.0945 Gramm & 0.3493 Gramm \\
\hline 8. März 1871 & 133 & 0.0597 & 0.4488 \\
\hline 1. Juli 1871 & 475 & 0.13787 & 0.29025 \\
\hline
\end{tabular}

Aus der Tabelle kann man entnehmen, dass das Maximum des Arsenikgebalts der Leber wenig von der von Dr. Geoghegan angegebenen Arsenikmenge in der Leber abweicht.

Existirt die Gesetzmässigkeit wirklich, so bätte die quantitative Bestimmung des Arsenik in der Menschenleber in forensisch-medicinischer Beziehung ein gewisses Interesse. Bei der Durchsicht der angeführten Tabelle, wie überhaupt der Versuche mit Arsenigsäure-Anhydrid, wäre noch nothwendig folgenden Erscheinungen Aufmerksamkeit zu schenken: 1) Im 1. und 3. Versuch wurde die Section am Tage nach der Vergiftung vorgenommen, im 2. Versuch aber erst nach 3 Monaten; 2) der Tod trat annähernd gewöhnlich eine Stunde nach der Vergiftung ein; 3) die Leber war im zweiten Versuch vön einem jungen Hunde.

Auf Grundlage dieser und anderer von mir erlangter Thatsachen, glaube ich mit einiger Wahrscheinlichkeit behaupten zu können, dass die in der Leber mit Arsenigsäure-Anhydrid vergifteter Thiere gefundene Arsenikmenge nicht besonders von der Grösse der Leber noch von der Zeit, während welcher der Arsenik im Organismus verweilte, beeinflusst wird.

Das Angefïhrte berechtigt zu folgendem Resumé.

1) Der Löslichkeitsgrad des Arsenigsäure-Anhydrids hängt von verschiedenen Umständen ab: von der Form des ArsenigsäureAnhydrids, von der Temperatur des Wassers, von der Dauer des Kochens und von der Dauer der Abkühlung der Lösung.

2) Das Arsenigsäure-Anhydrid geht im Organismus nicht in Arsensäure über, wenigstens kann man im Blute der mit ersterem Stoff vergifteten-Thiere denselben als solchen d. h. als eine arsenigsäure und nicht als arsensaure Verbindung nachweisen.

3) Kocht man Hühnereiweiss mit einer Lösung von Arsenig= 
säure-Anhydrid oder Arsensäure, so kann nicht behauptet werden, dass der eine Körper in den anderen überginge $d$. $h$. dass das Arsenigsäure-Anhydrid in Arsensäure oder umgekebrt, verwandelt würde.

4) Das Hühnereiweiss gerinnt beim Kochen mit ArsenigsäureAnhydrid, wie gewöhnlich beim Kochen ohne $\mathrm{As}_{2} \mathrm{O}_{3}$. Das Coagulum löst sich nicht durch Wasserzusatz und Kochen oder durch Aethylalkohol (78 nach Tralles) und Kochen.

Ganz anders verhält sich die Arsensäure zum Hühnereiweiss. Kocht man Hühnereiweiss mit einer wässrigen Lösung oder mit dem Pulver von Arsensäure, so erhält man nicht Gerinnung, sondern es bildet sich eine geléeartige Masse, welche sich vollständig beim Kochen nach Zusatz von starkem Aethylalkohol, oder auch nach Wasserzusatz löst. Diese geléeartige Masse ist eine Eiweissverbindung des Arsenik, wahrscheinlich Acidalbumin. Diese berïcksichtigend, kann ich mich nicht mit den Schlussfolgerungen von Prof. Binz und $\mathrm{Schulz}$ ), ungeachtet ihrer interessanten Untersuchungen, für einverstanden erklären; eher könnte ich mich der Theorie Liebig's anschliessen, dass die Arsenikwirkung auf den thierischen Organismus in der Bildung einer Eiweissverbindung des Arsenik besteht. In dieser Meinung werde ich noch dadurch bestärkt, dass ich bei der Wiederholung der von Binz beschriebenen, sich auf den Uebergang von Arsensäure, beim Vermischen mit Hïhnereiweiss, in Arsenigsäure-Anhydrid beziehenden Versuche, nach seiner Methode, ein negatives Resultat erhielt. Geben wir sogar zu, dass Arsenigsäure-Anhydrid nach Einwirkung der Arsensäure auf das Hühnereiweiss, bei der Binz'schen Bearbeitungsweise, auftritt, so geschieht dieses doch in äussert geringer Menge. Wenn solches auch im Organismus vor sich gehen könnte, sind aber hierfür doch noch strenge Beweise beizubringen; vorläufig jedoch, nach meinen Versuchen zu urtheilen, dürfte dieses nicht angenommen werden können.

5) Die Zersetzung der Cadaver der Thiere verlangsamt sich nach toxischen Arsenikgaben.

6) Die Arsenikanhäufung in der Leber geschieht mit gewisser Gesetzmässigkeit, wie es schon Geoghegan angiebt.

7) $\mathrm{As}^{2} \mathrm{O}^{3}$ bewirkt, dass defibrinirtes Frosch- oder Säugethier-

1) I. c. 
Beiträge z. Lehre v. d. Arsenikwirk. auf d. thierischen Organismus. 347

blut, sowohl in Bezug auf die Farbe, wie anf die Form der rothen Blutkörperchen, lange unverändert bleibt; während ebensolehes Blut durch die Wirkung von Arsensäure, $\mathrm{H}_{3} \mathrm{AsO}_{4}$, schnell dunkel wird und die rothen Blutkörperchen in der Form und Grösse eine Veränderung erleiden.

8) Das Arsenigsäure-Anhydrid wirkt je nach der Gabe stark anf das Herz ein. Hierbei wird die Zahl, die Kraft und der Rhythmus der Herzcontractionen abgeändert. Als nächste Ursache möchte ich eine Wirkung des Arsenik erst auf den Hemmungsapparat und dann auf die automatischen Nervencentra des Herzens, wie auch auf die Muskeln dieses Organs annehmen. Das Athmen wird gewöhnlich früher eingestellt, als die Herzfunction aufhört.

9) Der Blutdruck sehwankt nach der Grösse der Dosis des Arsenigsäure-Anbydrids oder anderer Arsenverbindungen. Interessant ist die Erscheinung, dass der Blutdruck noch steigt, während die Athmung eingestellt ist und das Herz nur noch schwach und selten schlägt, oder schon seine Bewegungen aufgebört haben.

10) Der Blutstrom wird anfangs durch die Arsenwirkung beschleunigt, hauptsächlich jedoch bei kleinen Gaben. Grosse Dosen führen anfangs eine Beschleunigung und darnach eine Verlangsamung des Blutstroms herbei.

(Physiol. Laboratorium der Thierarzneischule zu Bern.)

\section{Zur Beziehung von Leitungs- und Erregungsver. mögen der Nervenfaser.}

Von

Med. Dr. J. Szpilman und Prof. Dr. B. Luchsinger.

Leicht genug wird man versucht sein, die Leitungsfähigkeit der Nervenfaser in enge Beziehung zu setzen mit deren Erregungsfähigkeit. Wenn nun jedes erregte Nervenelement erregend auf die benachbarten einwirkt - eine gewiss allereinfachste Vorstellung 

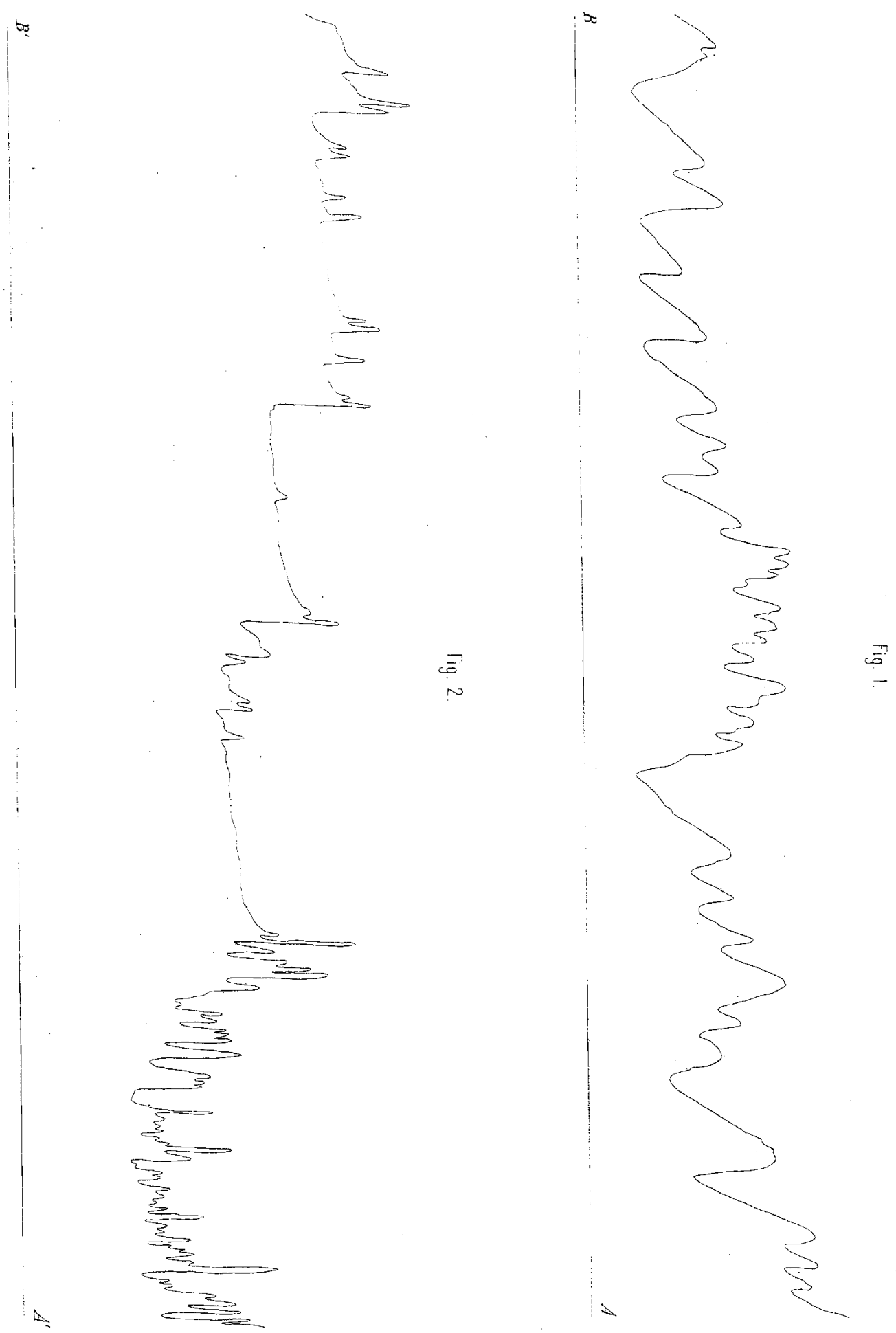